\title{
Cardiac regeneration validated
}

To the Editor:

In a recent review published in Nature Biotechnology, Naumova et al. ${ }^{1}$ repeat previously articulated concerns ${ }^{2}$ about the validity of contrast-enhanced magnetic resonance imaging (MRI) in characterizing myocardial regeneration after cell therapy. The authors raise theoretical concerns that cell administration may promote changes in myocardial vasculature (i.e., a decrease in vascular permeability resulting in reduced gadolinium (Gd)-contrast extravasation or an increase in lymphatic drainage leading to accelerated Gd-contrast washout), which would compromise the ability of contrastenhanced MRI to accurately measure changes in scarred and viable myocardium after cell therapy. In addition, Naumova et al. ${ }^{1}$ point out (correctly) that MRI cannot distinguish between myocardial hypertrophy and hyperplasia; thus, the increase in viable myocardial mass observed in patients treated with cardiosphere-derived cells (CDCs) could result from hypertrophy of pre-existing cardiomyocytes rather than generation of new myocardium. The concerns are not inconsequential, as they tend to undermine the conclusion that therapeutic regeneration occurred in patients treated with CDCs in the randomized Cardiosphere-derived Autologous Stem Cells to Reverse Ventricular Dysfunction (CADUCEUS) trial ${ }^{3,4}$.

We have addressed these concerns experimentally ${ }^{5}$ in work not cited in Naumova et al. ${ }^{1}$. Using a porcine model of ischemic cardiomyopathy that was designed to mimic the protocol in the CADUCEUS trial, we provided direct histological confirmation of the validity of MRI measurements of scar size, scar mass and viable mass. Areas classified as either scarred or viable by MRI agreed precisely with the quantification derived from corresponding tissue sections. In addition, we demonstrated that $\mathrm{CDC}$ administration does not alter Gd-contrast kinetics (thus excluding the theoretical possibility of reduced contrast extravasation or accelerated contrast washout), and these findings are consistent with the observed lack of changes in vascular density or architecture. Finally, histological measurement of myocyte size excluded myocyte hypertrophy as a contributor to the increase in viable myocardium observed after CDC therapy.

In conclusion, we have validated the fidelity of contrast-enhanced MRI to distinguish and accurately quantify scarred and viable myocardium after cell therapy, supporting its utility for assessing dynamic changes in the infarct and monitoring regenerative efficacy of cell-based therapeutic interventions.

COMPETING FINANCIAL INTERESTS

The authors declare competing financial interests: details are available in the online version of the paper (doi:10.1038/nbt.3254).

\section{Konstantinos Malliaras ${ }^{1}$ \& Eduardo Marbán ${ }^{1}$ \\ ${ }^{1}$ Cedars-Sinai Heart Institute, Los Angeles, California, USA. \\ e-mail:eduardo.marban@csmc.edu \\ 1. Naumova, A.V., Modo, M., Moore, A., Murry, C.E. \& Frank, J.A. Nat. Biotechnol. 32, 804-818 (2014). \\ 2. Chin, M.T. \& Murry, C.E. Regen. Med. 7, 623-625 (2012). \\ 3. Makkar, R.R. et al. Lancet 379, 895-904 (2012). \\ 4. Malliaras, K. et al. J. Am. Coll. Cardiol. 63, 110-122 (2014). \\ 5. Malliaras, K. et al. Circulation 128, 2764-2775 (2013).}

\section{Naumova et al. reply:}

We appreciate the comments from Malliaras and Marbán ${ }^{1}$ describing their work on developing imaging markers of therapeutic efficacy $^{2}$ that was not mentioned in our review $^{3}$. Owing to the page limitation, we were not able to cite all the literature published on this topic. We of course agree that magnetic resonance imaging (MRI) is a valuable technology for evaluating the efficacy of cell therapy, heart contractility, myofiber architecture and infarct size, especially in large-animal models. However, validation of imaging markers requires control measurements, especially in the assessment of gadolinium-contrast kinetics. As these measurements were reported only for cell-treated animals ${ }^{2}$, it remains unclear whether treatment changes the clearance rate in the scar. Decreases in vascular permeability and reduced extravasation after stem cell transplantation have been reported in controlled studies ${ }^{4,5}$, indicating that there is experimental evidence rather than a mere theoretical concern regarding these potential confounding factors.

Nevertheless, we agree that there was a good correlation between scar size as determined by MRI and histology at the end of the study by Malliaras et al. ${ }^{2}$, suggesting that cellinduced scar shrinkage may indeed be a real phenomenon.

The claimed growth of 10-15 g of new myocardium ${ }^{2}$ was not as well substantiated. Although there was not an increase in myocyte diameter, cell length was not measured and morphometric estimates of cell number were not obtained. Furthermore, although cell cycle activity was increased threefold, the overall rates were quite low (6 proliferative cells per square millimeter). It remains unclear how such a low rate of proliferation could lead to such a large increase in myocardial mass. Considering the heart's wellknown proclivity to generate polyploid cardiomyocytes without cell division, a more conservative interpretation of the data would seem prudent until more definitive evidence is available. Still, if the heart did undergo replacement of infarct mass by new cardiomyocytes, it would probably manifest the changes the authors reported by MRI. Additionally, if 10-15 g of new myocardium really were generated through cell division, this should be easy to show by conventional histology techniques, such as BrdU pulselabeling or cumulative labeling experiments. We look forward to seeing such studies in the future. In the meantime, we are intrigued by the potency of cardiospherederived cells to effect cardiac repair but remain cautious about their proposed mechanisms of action.

\section{Anna VNaumova ${ }^{1-3}$, Michel Modo ${ }^{4-7}$, Anna Moore ${ }^{8}$, Joseph A. Frank ${ }^{9,10}$ \& Charles E Murry $y^{2,3,11-13}$}

${ }^{1}$ Department of Radiology, University of Washington, Seattle, Washington, USA. ${ }^{2}$ Center for Cardiovascular Biology, University of Washington, Seattle, Washington, USA. ${ }^{3}$ Institute for Stem Cell and Regenerative Medicine, University of Washington, Seattle, Washington, USA. ${ }^{4}$ Mc Gowan Institute for Regenerative Medicine, University of Pittsburgh, Pittsburgh, Pennsylvania, USA. ${ }^{5}$ Centre for the Neural Basis of Cognition, University of Pittsburgh, Pittsburgh, Pennsylvania, USA. ${ }^{6}$ Department of Radiology, University of Pittsburgh, Pittsburgh, Pennsylvania, USA. ${ }^{7}$ Department of Bioengineering, University of Pittsburgh, Pittsburgh, Pennsylvania, USA.

${ }^{8}$ Athinoula A. Martinos Center for Biomedical Imaging, Department of Radiology, Massachusetts General Hospital, Charlestown, Massachusetts, USA. ${ }^{9}$ Radiology and Imaging Sciences, Clinical, National Institutes of Health, Bethesda, Maryland, USA. ${ }^{10}$ National Institutes of Biomedical Imaging and Bioengineering, National Institutes of Health Bethesda, Maryland, USA. ${ }^{11}$ Department of Pathology, University of Washington, Seattle, Washington, USA. ${ }^{12}$ Department of Bioengineering, University of Washington, Seattle, Washington, USA. ${ }^{13}$ Department of Medicine/ Cardiology, University of Washington, Seattle, Washington, USA.

e-mail:murry@uw.edu.

1. Malliaras, K. \& Marbán, E. Nat. Biotechnol. 33, 587 (2015).

2. Malliaras, K. et al. Circulation 128, 2764-2775 (2013)

3. Naumova, A.V., Modo, M., Moore, A., Murry, C.E. \& Frank, J.A. et al. Nat. Biotechnol. 32, 804-818 (2014).

4. Pati, S. et al. Stem Cells Dev. 20, 89-101 (2011)

5. Zhao, Y.D. et al. Circ. Res. 105, 696-704 (2009). 